\title{
Shadow imaging in bubbly gas-liquid two-phase flow in porous structures
}

\author{
Marco Altheimer ${ }^{1}$ Richard Häfeli ${ }^{1}$ Carmen Wälchli ${ }^{1}$ Philipp Rudolf von Rohr ${ }^{1}$
}

Received: 19 January 2015 / Revised: 30 July 2015 / Accepted: 9 August 2015 / Published online: 7 September 2015

(C) Springer-Verlag Berlin Heidelberg 2015

\begin{abstract}
Shadow imaging is used for the investigation of bubbly gas-liquid two-phase flow in a porous structure. The porous structure is made of Somos ${ }^{\circledR}$ WaterShed XC 11122, a clear epoxy resin used in rapid prototyping. Optical access is provided by using an aqueous solution of sodium iodide and zinc iodide having the same refractive index as the structure material $(n=1.515)$. Nitrogen is injected into the continuous phase at volumetric transport fractions in the range of $\dot{\varepsilon}=2.4-4.1 \%$ resulting in a hold-up of $\varepsilon=0.94-2.17 \%$. The obtained images of overlapping bubble shadows are processed to measure the bubble dimensions. Therefore, a new processing sequence is developed to determine bubble dimensions from overlapping bubble shadows by ellipse fitting. The accuracy of the bubble detection and sizing routine is assessed processing synthetic images. It is shown that the developed technique is suitable for volumetric two-phase flow measurements. Important global quantities such as gas hold-up and total interfacial area can be measured with only one camera. Operation parameters for gas-liquid two-phase flows are determined to improve mass and heat transfer between the phases.
\end{abstract}

\section{List of symbols}

\section{Roman symbols}

A Area $\left(\mathrm{mm}^{2}\right)$

$C \quad$ Constant $\left((\sqrt{\operatorname{pix}} s)^{-1}\right)$

$D \quad$ Sum of algebraic distances (-)

Marco Altheimer

altheimer@ipe.mavt.ethz.ch

Philipp Rudolf von Rohr

vonrohr@ipe.mavt.ethz.ch

1 Institute of Process Engineering, ETH Zürich,

Sonneggstrasse 3, 8092 Zürich, Switzerland
$F \quad$ Frequency threshold $(\mathrm{Hz})$

$F \quad$ Function (-)

$L \quad$ Length (mm)

Re Reynolds number (-)

$V \quad$ Volume $\left(\mathrm{mm}^{3}\right)$

$a-f \quad$ Coefficients (-)

a Coefficients vector $(-)$

a Major semi-axis (mm)

$b \quad$ Minor semi-axis (mm)

c Minor semi-axis (mm)

$d \quad$ Diameter (mm)

$e \quad$ Numerical eccentricity (-)

$h \quad$ Height (mm)

$k \quad$ Curvature (1/pix)

$k \quad$ Number of drawings (-)

$n \quad$ Refractive index (-)

p Sample size (-)

$s \quad$ Slip (-)

$s \quad$ Perimeter length (pix)

$v \quad$ Velocity $(\mathrm{m} / \mathrm{s})$

x Variables vector (-)

$x, y \quad$ Positions (pix)

$x^{\prime}, y^{\prime} \quad$ First-order derivatives (-)

$x^{\prime \prime}, y^{\prime \prime} \quad$ Second-order derivatives (1/pix)

\section{Greek letters}

$\epsilon \quad$ Relative error (\%)

$\varepsilon \quad$ Porosity (-)

$\varepsilon \quad$ Hold-up (\%)

$\dot{\varepsilon} \quad$ Volumetric transport fraction (\%)

$\eta \quad$ Dynamic viscosity $(\mathrm{kg} /(\mathrm{m} \mathrm{s}))$

$\rho \quad$ Density $\left(\mathrm{kg} / \mathrm{m}^{3}\right)$

$\sigma \quad$ Coefficient of variation (-)

$\sigma \quad$ Surface tension $(\mathrm{mN} / \mathrm{m})$

$\sigma \quad$ Standard deviation (-) 


$\begin{array}{ll}\text { Sub- and } & \text { superscripts } \\ 32 & \text { Sauter mean } \\ \mathrm{a} & \text { Major semi-axis } \\ \mathrm{b} & \text { Bubble } \\ \mathrm{c} & \text { Cell } \\ \mathrm{e} & \text { Edging } \\ \mathrm{h} & \text { Hydraulic } \\ \text { int } & \text { Interstitial } \\ \mathrm{l} & \text { Liquid } \\ \text { mean } & \text { Arithmetic mean } \\ \text { min } & \text { Minimum } \\ \text { o } & \text { Oblate } \\ \text { p } & \text { Pore } \\ \text { pipe } & \text { Pipe } \\ \text { pr } & \text { Prolate } \\ \text { PU } & \text { Periodic unit } \\ \text { sp } & \text { Spherical }\end{array}$

$\begin{array}{ll}\text { Abbreviations } \\ \text { b/w } & \text { Black and white } \\ \text { CAD } & \text { Computer-aided design } \\ \text { FFT } & \text { Fast Fourier transform } \\ \text { LED } & \text { Light-emitting diode } \\ \text { PIV } & \text { Particle image velocimetry } \\ \text { PMMA } & \text { Polymethyl methacrylate } \\ \text { PTV } & \text { Particle tracking velocimetry } \\ \text { PU } & \text { Periodic unit } \\ \text { P\&ID } & \text { Process and instrumentation diagram }\end{array}$

\section{Introduction}

In the past, bubbly gas-liquid two-phase flows in rectangular channels, pipes and bubble columns have been investigated very often to determine the size, shape, velocity, hold-up, coalescence and break-up of bubbles as well as the turbulent statistics of the continuous liquid phase. Therefore, a considerable number of intrusive and non-intrusive (Chaouki et al. 1997) measurement techniques have been developed. For the determination of gas-liquid two-phase flow characteristics, intrusive measurement probes, such as hot-film anemometer (e.g., Garnier et al. 2002; Lance and Bataille 1991) or conductivity probes (e.g., Hibiki and Ishii 1999; Wu and Ishii 1999) as well as non-intrusive techniques such as laser Doppler anemometry (e.g., Lance and Bataille 1991; So et al. 2002) or ultrasonic Doppler technique (e.g., Murakawa et al. 2005), have been used. Additionally, a wide choice of optical techniques was presented in the literature, where cameras are used to acquire images showing bubbles or tracer particles.

\subsection{Optical techniques with cameras}

Another class of non-intrusive measurement techniques is formed by optical arrangements using cameras for bubble detection and sizing. It can be differentiated between measurement techniques capturing only single bubbles or cut sections of the flow, measurement techniques capturing the whole volume but excluding out-of-focus bubbles or overlapping bubble shadows in the images, and techniques capturing the whole volume and evaluating all bubbles in the flow. In the following paragraphs, the literature is reviewed for above-mentioned techniques.

In a study of slug flows, Nogueira et al. (2003) investigated single Taylor bubbles rising in a vertical pipe of stagnant liquid. They simultaneously applied two-dimensional particle image velocimetry (PIV) and a pulsed shadow technique to determine the flow field and the bubble shape of single bubbles. Single bubbles in a simple shear layer have been investigated by Fujiwara et al. (2004). The flow structure in the vicinity of the bubble is determined by PIV with fluorescent particles, and the bubble deformation is observed from two angles using shadow imaging. Kim et al. (2012) analyzed the spatial and temporal structure of air bubble-driven turbulent water flows in a cylindrical tank. They used a combination of volumetric illumination from the top and a vertical laser plane for PIV with fluorescent particles. Densely binding bubble clusters arranged in one plane have been investigated by Ma et al. (2014). They used a classical shadow imaging technique with background illumination to accurately measure the size of small $(<1 \mathrm{~mm})$ spherical bubbles.

Bubble swarms which are not only arranged in one plane layer (Ma et al. 2014), but free to move in a volume have been investigated quite often. Sathe et al. (2010) focused on size, shape, velocity and acceleration measurements of bubbles using PIV and shadow imaging. They investigated twophase flows with bubbles of a wide size range $(0.1-15 \mathrm{~mm})$ at a hold-up of $5 \%$. Experiments were conducted in a narrow rectangular column to lower/eliminate overlapping of bubble shadows. Overlapping of bubble shadows can also be eliminated by using a laser plane instead of background illumination, see Bröder and Sommerfeld (2002). They investigated two-phase flows in a bubble column with a mean bubble diameter of 0.5-4 mm and hold-ups of 0.5-19 \% using pulsed-light velocimetry and fluorescent seeding particles. Instantaneous velocity fields of both phases can be obtained up to a hold-up of $6 \%$. Similar to this, Lindken and Merzkirch (2000) investigated bubbles rising in water. Instead of one vertical laser plane, they illuminated a vertical plane for PIV and a horizontal plane for bubble recognition and sizing. Again, the planar illumination eliminates the problem of overlapping bubble shadows. 
This problem of overlapping shadows must be solved for measuring techniques capturing volumetric information (e.g., shadow imaging). Therefore, different setups or filter functions are applicable. Colella et al. (1999) analyzed breakage and coalescence phenomena in three different bubble columns by manually tracing in-focus bubbles. The size was determined by automatic ellipse fitting assuming oblate ellipses for the estimation of the third axis. Bröder and Sommerfeld (2007) studied a turbulent bubbly flow in a bubble column at hold-ups of $0.5-5 \%$ and mean bubble diameters of $2-4 \mathrm{~mm}$ using PIV and particle tracking velocimetry (PTV). A CCD camera with macro-optics was used, and the gradient of gray values was analyzed to distinguish between in- and out-of-focus particles. The bubble contour was determined by Sobel filtering and spline interpolation. Similarly, Castanet et al. (2013) measured droplet sizes of impacting droplets on to a wall heated above the Leidenfrost temperature. Separation of overlapping, sometimes strongly deformed, droplet shadows was carried out by the use of topographic information in the gray-level image or by analyzing the curvature of the object. Such curvature analysis was also used by Honkanen et al. (2011) to individually recognize overlapping bubble shadows in microscale bubbly flows. Therefore, the perimeter curvature was analyzed, and a so-called breakline method was applied. Beside this, a correlation-based procedure was presented by Akhmetbekov et al. (2010) to identify overlapping bubble shadows from planar measurements. They stated that the procedure can be easily applied to shadow photography images by including a derivative filter (e.g., Sobel transform). A limit of $5 \%$ gas hold-up is given where severely overlapping bubble shadows are expected. If none of the above-described methods deliver satisfying results, two authors suggested to remove overlapping bubble shadows from statistical computations (Kitagawa et al. 2005; Rodríguez-Rodríguez et al. 2003).

A full picture of the bubble distribution and flow characteristics in bubbly two-phase flow can be obtained (up to a critical hold-up) by three-dimensional optical measurement. Belden et al. (2012) presented a threedimensional synthetic aperture imaging process for resolving three-dimensional bubble fields. They state that their procedure is suitable to investigate optically dense multiphase flows capturing entirely in-focus images from multiple viewpoints. A refocusing algorithm is then generating a synthetic focal stack to isolate the depth of bubbles. Murai et al. (2001) used stereoscopic image processing to capture the shadows of the bubbles from two locations. To reconstruct the image, the bubble-existence probability function satisfying the two distributions of the two projection void fractions is determined by a logical method. Generally, it must be mentioned that such three-dimensional measurements are costly (several cameras needed) and computationally expensive.

\subsection{Complex geometries with index matching}

Beside above-mentioned geometries, porous structures, fixed beds or fluidized beds were also investigated in the past. Optical access was provided by index matching between the structure material and the liquid phase. Chen and Fan (1992) investigated the flow structure in gas-liquid-solid fluidized beds by matching the refractive index of the fluid to the solid. Northrup et al. (1993) measured the interstitial velocity field variations in a porous medium. Therefore, they used fluorescent particles for PIV and matched the liquid phase to the porous medium test bed made of polymethylmethacrylate (PMMA). Another porous structure was investigated by Butscher et al. (2012). The structure was made of an epoxy resin, and anisole was used as liquid phase. Investigations of two-phase flows with index matching was also done in the past. Kong et al. (2011) visualized air injection in a liquid-saturated porous medium. They illuminated plane wise by a laser using a rotating prism for time-resolved measurements.

The present work focuses on the shadow imaging of bubbles inside a designed porous structure. Index matching between the structure material and liquid phase is carried out to provide optical access. A novel bubble detection routine is developed for the detection and separation of overlapping bubble shadows. This new routine allows size measurements of all bubbles enabling the determination of global quantities, such as the hold-up, interfacial area and slip. Previous studies, where only in-focus bubbles are taken into account and all other shadows with blurred contours are discarded, focused on size and velocity measurements. Bröder and Sommerfeld (2007), for example, determined the bubble size based on edge detection and spline interpolation of the in-focus bubbles. Honkanen et al. (2005) measured the size of in-focus bubbles applying a sequence of image thresholding and ellipse fitting. Both experiments were conducted in bubble columns with rather large depth, where it would be difficult to determine all bubbles from overlapping shadows. Hirschberg et al. (2009) conducted droplet size measurements at the outlet of a static mixer. To simplify the droplet detection, they used an optical flow cell with a narrow gap. This limited the number of overlapping droplets and allowed a simple bubble sizing by using the software BubbleCount developed at TU Berlin. 
In this work, we focused on the technique developed by Honkanen et al. (2005). The principle is extended to allow overlapping bubble shadows to be separated and measured individually. This allows the direct determination of global quantities. The new routine is verified using synthetic images of bubbles.

\section{Experiments}

\subsection{Setup}

In Fig. 1, the process and instrumentation diagram (P\&ID) of the setup is depicted. The liquid phase is contained in a storage tank (1) which is also serving as phase separator. A VerderCatag VGS200 gear pump (2) is recirculating the fluid through the system and back to the storage tank. A Bronkhorst M55-AGD-22-O-S mass flow controller (3) with an accuracy of $0.2 \%$ is used to control the mass flow rate and measure the density of the continuous phase. The temperature at the inlet (4) and outlet (6) is measured and a heat exchanger (5) is placed in front of the test section to ensure a constant temperature. A calming section of $1 \mathrm{~m}$ length (8) is used to ensure fully developed flow before entering the porous structure (9). Right in front of the porous structure (9), nitrogen at 7 bar is injected into the continuous phase through a single nozzle of $1 \mathrm{~mm}$ in diameter. Therefore, pressurized nitrogen is fed from the bottle (11), and the volume flow is controlled by a Brooks $5850 \mathrm{~S}$ mass flow controller (12) with an accuracy of $1 \%$. A relief valve (13) is mounted on top of the storage tank to remove the excess nitrogen and ensure that there is no oxygen in the system.

\subsection{Measurement equipment}

The measurement equipment consists of only four parts: camera, background illumination, synchronizer and computer. The synchronizer controls the camera shutter and the LED (HPLS-36DD7500) used for background illumination. A good introduction of LED heads and pulsed operation for flow velocimetry is presented in Willert et al. (2010). For homogeneous background illumination, the LED is equipped with a diffuser plate made of PMMA. A pco.1600 camera with a resolution of $1600 \times 1200$ pixel is used for image acquisition. In Fig. 2, the arrangement of camera and background illumination around the structure is depicted schematically.

\subsection{Porous structure}

\subsubsection{Geometry}

The patented porous structure (Hutter et al. 2010), already used in other experiments in our laboratory (e.g., Häfeli et al. 2013; Hutter et al. 2011), is geometrically defined and represents the negative pattern of tetrahedrally arranged overlapping spheres. Three stacks of spheres $(A-B-C)$ form one periodic unit $(\mathrm{PU})$. An auxiliary view of one periodic unit is shown in Fig. 3a.

The negative of a sphere is called cell. At the points of overlapping, the cell is connected to other cells. Each cell in the middle of the structure is connected to twelve neighboring cells. The connections are called pores and define the smallest passage the fluid has to

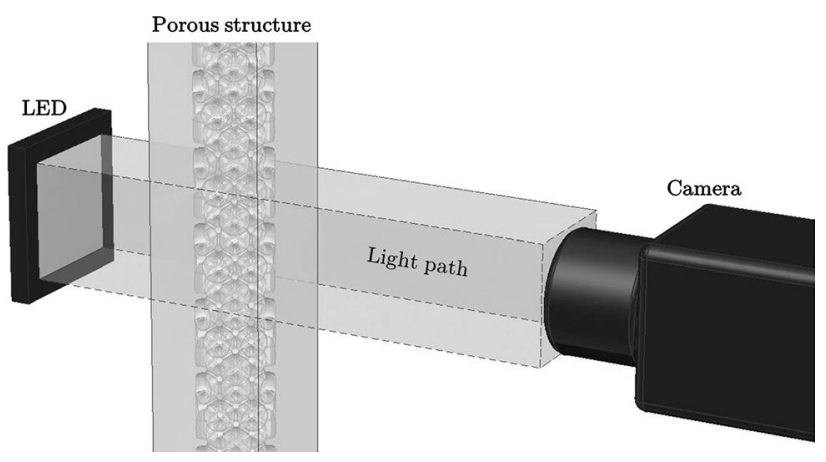

Fig. 2 Optical measurement equipment and arrangement

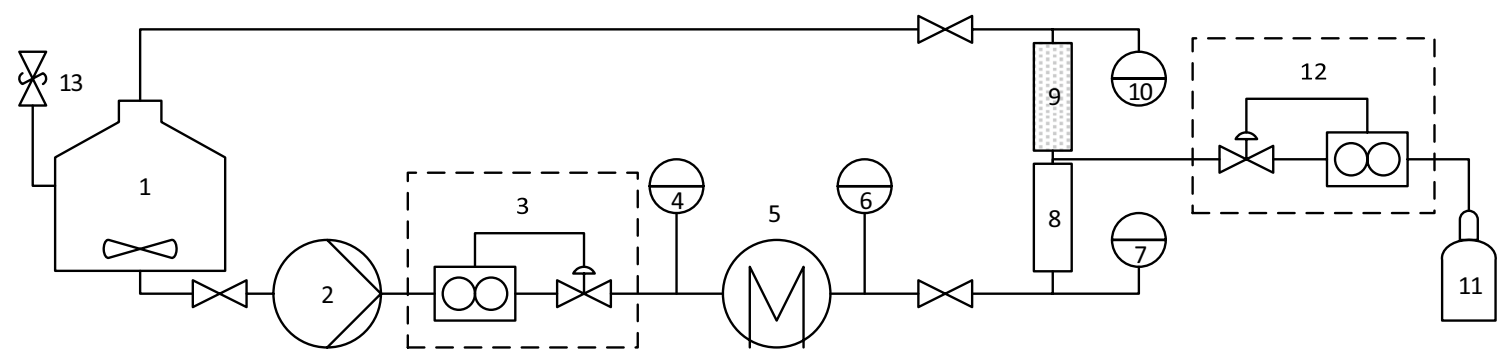

Fig. 1 P\&ID of the experimental setup excluding the optical part (background illumination and camera) 
go through. In Fig. 3b, the cells and pores are depicted in a cut-sectional view of stack A.

Due to the very little overlapping of cells, the pores connecting the cells have a very sharp circumferential edge. This edge cannot be accurately produced by rapid prototyping (Hutter et al. 2011). Therefore, the edge is rounded off in the computer-aided design (CAD) model. In Fig. 3c, the edging is schematically shown.

The structure fits inside a pipe of $20 \mathrm{~mm}$ in diameter. To reduce dead volumes, the outermost cells in stacks B and $\mathrm{C}$ are removed from the structure as there is nearly no flow inside these cells. In Fig. 3d, the removed cells are depicted with dashed lines. The removal of the outermost cells decreases the porosity of the structure to $70.35 \%$. In previous investigations, the outermost cells were not removed and the porosity was $78.7 \%$ (Häfeli et al. 2014). All dimensions of the structure are given in Table 1. For a better comparison between experiments and the literature, the Reynolds number is calculated (Eq. 1) based on the hydraulic diameter and the interstitial velocity of the liquid phase in the structure $(\dot{\varepsilon}=0 \%)$. The hydraulic diameter $d_{h}$ is determined to be $6.234 \mathrm{~mm}$.

$R e=\frac{v_{\text {int }, 1} d_{h} \rho_{l}}{\eta_{l}}$

where $v_{\text {int, } 1}(\mathrm{~m} / \mathrm{s})$ : interstitial velocity of the liquid phase in the structure $(\dot{\varepsilon}=0 \%), d_{h}(\mathrm{~mm})$ : hydraulic diameter, $\rho_{l}\left(\mathrm{~kg} / \mathrm{m}^{3}\right)$ : liquid density and $\eta_{l}(\mathrm{~kg} /(\mathrm{m} \mathrm{s}))$ : liquid viscosity. (a)

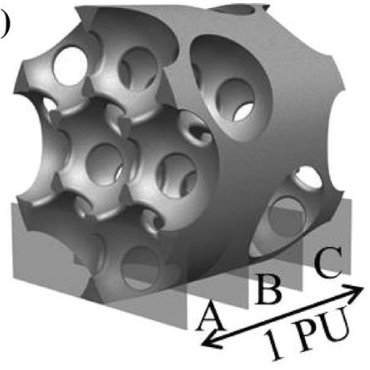

(c)

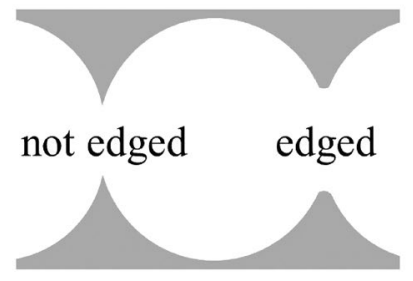

(b)

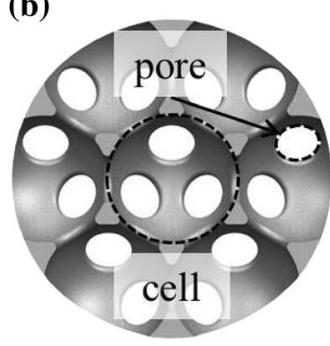

(d)

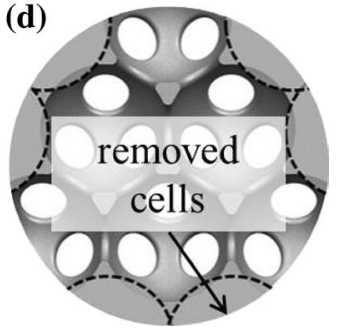

Fig. 3 Porous structure: a auxiliary view of one periodic unit, $\mathbf{b}$ stack A with a cell and pore indicated by dashed lines, comparison: not edged-edged pore, $\mathbf{d}$ ghosts of removed cells at the pipe wall indicated by dashed lines
Table 1 Dimensions of the porous structure

\begin{tabular}{lllll}
\hline$d_{c}(\mathrm{~mm})$ & $d_{p}(\mathrm{~mm})$ & $r_{e}(\mathrm{~mm})$ & $L_{c}(\mathrm{~mm})$ & $L_{P U}(\mathrm{~mm})$ \\
\hline 7.94 & 2.88 & 0.34 & 7.86 & 19.25 \\
\hline
\end{tabular}

\subsubsection{Material}

The porous structure is made of Somos ${ }^{\circledR}$ WaterShed XC 11122, a low-viscosity liquid photopolymer (DSM 2014). The liquid photopolymer is processed by stereolithography, resulting in a strong, tough, water-resistant and nearly colorless material, ideal for optical experiments. The accuracy of the stereolithographic process is limited by the layer thickness of $0.15 \mathrm{~mm}$. Structure and pipe are produced in one piece.

Butscher et al. (2012) discovered that the orientation of the stereolithographic layers is influencing the optical access to the structure. Therefore, the layers should not be parallel to any orientation of light when working with a laser. For experiments with background illumination (diffuse light), no significant disturbance was recognized.

More important than the layering direction is the refractive index matching of structure material and fluid, see Sect. 2.4. In the specifications of Somos ${ }^{\circledR}$ WaterShed XC 11122 (DSM 2014), the refractive index $n$ is given to be in the range of 1.512 and 1.515 . In the present work, the upper limit of the refractive index given in the specifications (DSM 2014), $n=1.515$, is used for index matching. This decision was made due to the fact that for in-house refractive index measurements of solid materials with the Abbe refractometer from Carl Zeiss AG, a contact liquid of higher refractive index (monobromonaphthalene, $n=1.66$ ) is used. The contact liquid could also be absorbed by the structure and adulterate the results. In previous experiments, the refractive index of the WaterShed XC 11122 was determined to be 1.519 using the Abbe refractometer from Carl Zeiss AG (Häfeli et al. 2014). It shows that smaller variations of the refractive index do not drastically alter the matching quality.

\subsection{Fluids}

\subsubsection{Continuous liquid phase}

An aqueous salt solution of sodium iodide and zinc iodide, proposed by Häfeli et al. (2014), is used as continuous liquid phase. This solution allows an adaption of the refractive index by varying the salt concentration. Additionally, the structure material is chemically stable in this solution. The main problem of the proposed salt solution is the oxidation of zinc iodide and the associated discoloration of the liquid. Discoloration can be prevented by the addition of sodium 
thiosulfate $\left(\mathrm{Na}_{2} \mathrm{~S}_{2} \mathrm{O}_{3}\right)$, as proposed by Svensson and Rasmuson (2006) and Narrow et al. (2000). Nevertheless, the $\mathrm{Na}_{2} \mathrm{~S}_{2} \mathrm{O}_{3}$ does not prevent oxidation, and property changes are possible when the zinc iodide is oxidized. To overcome the problem with oxidation, the system was closed to the surrounding and flushed with nitrogen to prevent oxidation and discoloration.

A Haake viscosimeter VT550, a Bronkhorst massflow meter M55-AGD-22-O-S and a Krüss K100 tensiometer (Wilhelmy plate method) were used to measure the viscosity, density and surface tension, respectively. As mentioned in Sect. 2.3, the refractive index was matched to the upper limit of the refractive index given in the specifications of the structure material Somos ${ }^{\circledR}$ WaterShed XC 11122 (DSM 2014), $n=1.515$. Therefore, the composition proposed by Häfeli et al. (2014)—1000 mg H ${ }_{2} \mathrm{O}, 1400 \mathrm{mg} \mathrm{NaI}, 730 \mathrm{mg}$ $\mathrm{ZnI}_{2}$ - was slightly changed to $1000 \mathrm{mg} \mathrm{H}_{2} \mathrm{O}, 1400 \mathrm{mg}$ $\mathrm{NaI}, 680 \mathrm{mg} \mathrm{ZnI}$. An Abbe refractometer from Carl Zeiss AG was used to measure the refractive index. The fluid was tempered to $20{ }^{\circ} \mathrm{C}$ before entering the porous structure to eliminate property changes due to temperature variations. The most important fluid properties of both fluids are given in Table 2.

\subsubsection{Disperse gas phase}

Nitrogen serves as the disperse gas phase. It is the ideal choice in combination with the aqueous salt solution. Nitrogen is inert and prevents the salt solution from oxidation and discoloration. Additionally, nitrogen is absorbed only very little in water.

\section{Image processing}

The image processing is done in MATLAB ${ }^{\circledR}$. A routine is developed and adapted to this specific problem of bubbly flow inside the porous structure. The routine is capable of detecting and separating overlapping bubble shadows and allows the determination of global quantities, such as holdup or total interfacial area. Raw images showing the minimum and maximum hold-up and diameter are presented in Fig. 4.

Table 2 Fluid properties at $20^{\circ} \mathrm{C}$

\begin{tabular}{llll}
\hline Fluid & Density $\rho\left(\mathrm{kg} / \mathrm{m}^{3}\right)$ & $\begin{array}{l}\text { Viscosity } \eta \\
(\mathrm{kg} /(\mathrm{m} \mathrm{s}))\end{array}$ & $\begin{array}{l}\text { Surface tension } \sigma \\
(\mathrm{mN} / \mathrm{m})\end{array}$ \\
\hline Salt solution & 1995 & 0.00415 & 55 \\
Nitrogen & 1.251 & $1.8 \mathrm{E}-5$ & \\
\hline
\end{tabular}

\subsection{Image preparation}

In a first step, the background image is subtracted to remove static objects, such as the structure. Then, a gray threshold according to Otsus' method (Otsu 1975) is chosen for a black and white (b/w) image conversion. The $b / w$ image is showing black spots in the middle of the bubbles where light was shining through as it entered and left the bubble perpendicularly to the surface of the bubble. These spots are removed by filling the contours. Additionally, the contours are closed by disk-shaped elements to eliminate discontinuities in the bubble contour. Discontinuities are challenging for the following ellipse fitting and must be avoided. Problematic is that the closing can lead to shadows which are growing together. Nevertheless, this effect is no problem for the following ellipse fitting. Examples of the image preparation are given in Fig. 5.

\subsection{Boundary detection and segmentation}

The boundaries of the white objects in Fig. 5d are detected as exemplarily shown in Fig. 6a. A moving average filter is applied to the boundary before the gradients are calculated by a centered difference scheme. Using the obtained gradients, the curvature $k$ is determined by Eq. (2).

$k=\frac{x^{\prime} \cdot y^{\prime \prime}-y^{\prime} \cdot x^{\prime \prime}}{\left(x^{\prime 2}+y^{\prime 2}\right)^{(3 / 2)}}$

where $k(1 / \mathrm{pix})$ : boundary curvature, $x^{\prime}(-), x^{\prime \prime}(1 / \mathrm{pix})$, $y^{\prime}(-), y^{\prime \prime}$ (1/pix): first- and second-order derivatives at the corresponding coordinate points $(x, y)$ on the detected boundary. The derivatives are determined from 10 pixels in every coordinate direction.

Honkanen et al. (2005) presented a method to detect curvature peaks on the boundary of the objects. Therefore, they applied a fast Fourier transform (FFT) low-pass filter on the curvature and set an empirically defined frequency threshold,

$F=C s\left(\frac{10}{\sqrt{s+30}}\right)$

where $F(\mathrm{~Hz})$ : frequency threshold, $C\left((\sqrt{\mathrm{pix}} \mathrm{s})^{-1}\right)$ : constant and $s$ (pix): perimeter length. They also stated that the constant $C=0.16(\sqrt{\mathrm{pix}} \mathrm{s})^{-1}$ in Eq. (3) is suitable for noisy bubble images where a value of $0.3(\sqrt{\text { pix } s})^{-1}$ is suitable for simulated images having a low noise level. The constant was evaluated by manually checking obtained results. A new value of $C=0.1(\sqrt{\text { pix }})^{-1}$ was found to deliver the best results in this work. Figure $6 \mathrm{~b}$, c shows the unfiltered and filtered curvature plotted versus the perimeter length. A global curvature threshold of -0.31 was chosen to detect curvature peaks, and an example is given in Fig. 6d, corresponding to the curvature profile in Fig. 6c. In the last step, 

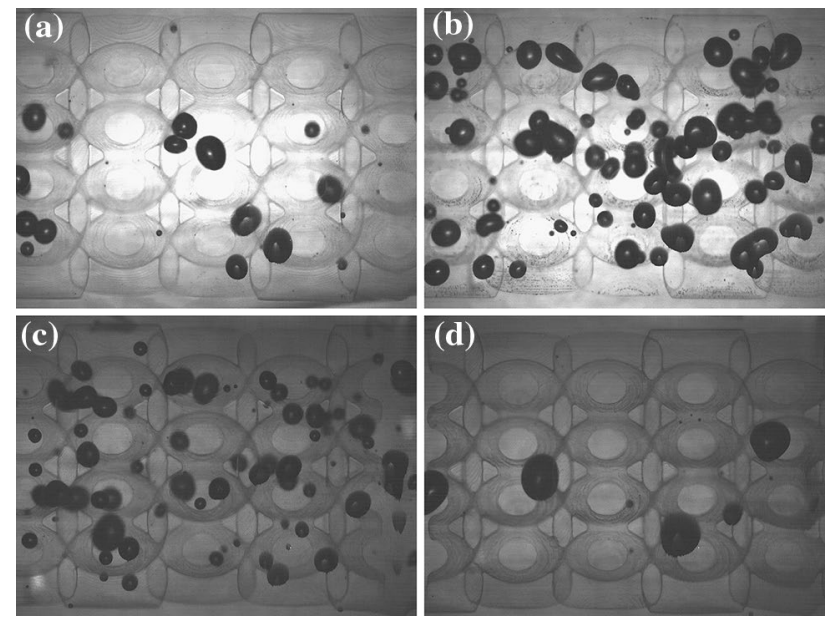

Fig. 4 Raw images of different experimental conditions: a minimum hold-up $(\operatorname{Re}=152, \dot{\varepsilon}=2.4 \%, \varepsilon=0.92 \%)$, b maximum hold-up $(\operatorname{Re}=285, \dot{\varepsilon}=4.1 \%, \varepsilon=2.17 \%)$, c minimum arithmetic mean diameter in PU $12\left(R e=285, \dot{\varepsilon}=3.2 \%, d_{\mathrm{b} \text {,mean }}=1.29 \mathrm{~mm}\right)$, d maximum arithmetic mean diameter in $\mathrm{PU} 1(R e=114$, $\dot{\varepsilon}=3.2 \%, d_{\mathrm{b} \text {, mean }}=2.10 \mathrm{~mm}$ )
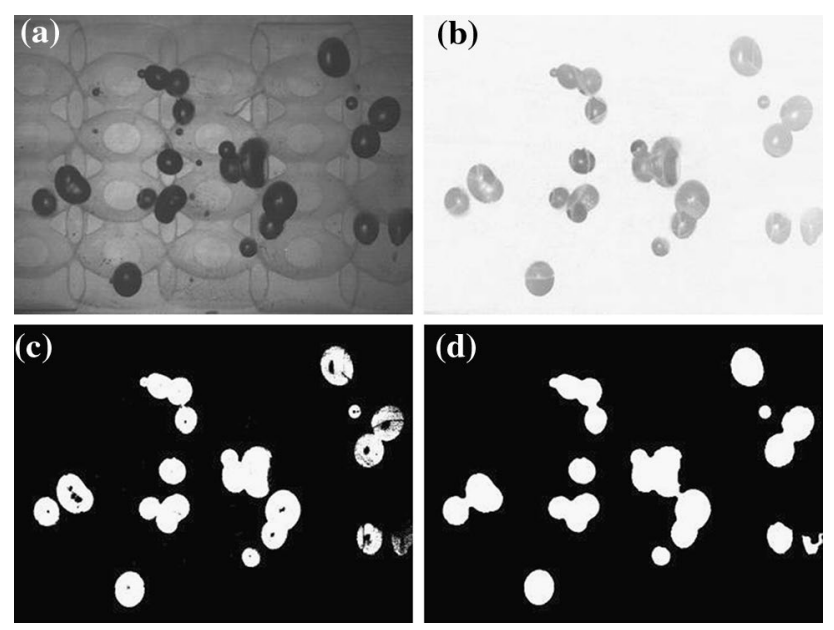

Fig. 5 Image preparation: a raw image, b background removed, c black and white image conversion, $\mathbf{d}$ contours closed and filled

the boundary is separated into segments between the peaks to prepare the images for ellipse fitting.

\subsection{Ellipse fitting}

The description of the shape regimes for bubbles and drops in unhindered gravitational motion through liquids was used to estimate the shape of the bubbles (Clift et al. 2005). It can be seen that the most deformed shape-for highest Reynolds and Eötvös numbers-is below the wobbling regime and rather in the ellipsoidal and spherical regime. Therefore, it was decided to fit ellipses along the boundary segments. The direct least-square fitting algorithm developed by Fitzgibbon et al. (1999) is used. They take a general conic described by an implicit second-order polynominal:

$F(\mathbf{a}, \mathbf{x})=a x^{2}+b x y+c y^{2}+d x+e y+f=0$

where

$\mathbf{a}=[a b c d e f]^{T}$

$\mathbf{x}=\left[x^{2} x y y^{2} x y 1\right]^{T}$.

$F\left(\mathbf{a}, \mathbf{x}_{\mathbf{i}}\right)$ is the distance of point $(x, y)$ to the conic $F(\mathbf{a}, \mathbf{x})=0$. To fit a general conic, the sum of squared algebraic distances must be minimized:

$D(\mathbf{a})=\sum_{i=1}^{N} F\left(\mathbf{x}_{\mathbf{i}}\right)^{2}$

Fitzgibbon et al. (1999) imposed an equality constraint $4 a c-b^{2}=1$ to force conics into ellipses while retaining the efficiency of the linear least-square problem. The proposed algorithm combines several features such as ellipsespecific fitting, high robustness to noise, high efficiency (not iterative) and easy implementation. Honkanen et al. (2005) also used this fitting algorithm for bubble detection delivering good results. The MATLAB ${ }^{\circledR}$ script provided by Fitzgibbon et al. (1999) was used and implemented into our routine.

Figure 7a shows the result obtained by fitting ellipses along the segments. It can be seen that one single bubble shadow may be separated into more than only one segment if it is overlapping with more than one other bubble shadow. Therefore, additional fitting criteria are needed.

At first, it is checked whether the content of the fitted ellipse is white. If there are more than $45 \%$ black pixel inside the ellipse, the ellipse is discarded. This is important to remove ellipses which are much larger than the real bubble.

The second criterion is merging overlapping ellipses. Round ellipses, where $b / a>0.65$, are merged if they are overlapping for more than $75 \%$ in the first iteration, meaning that the boundary segments of the two ellipses are collected in one boundary segment and a new ellipse is fitted based on that segment. This procedure is repeated three times, where in the first iteration, the overlap must be larger than $75 \%$ and in the other two iterations, the overlap must be larger than $80 \%$. Beside round ellipses, thin ellipses with $b / a<0.65$ are also merged, but they must only overlap for $30 \%$ in the first iteration and $55 \%$ in the next two iterations. This differentiation of round and thin ellipses is necessary as thin ellipses may be obtained for large overlapping bubble shadows with only short segments, as exemplarily shown in Fig. 7a, b. 
(a)

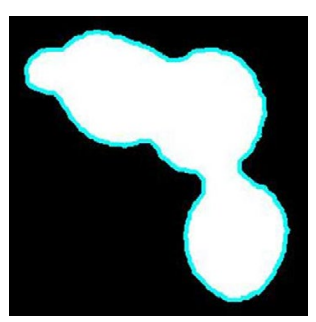

(c)

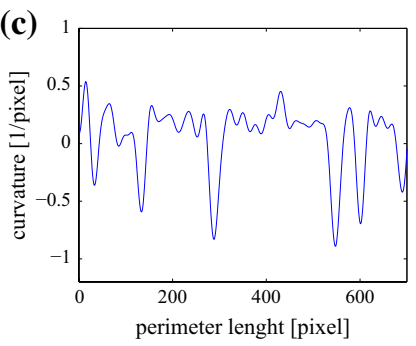

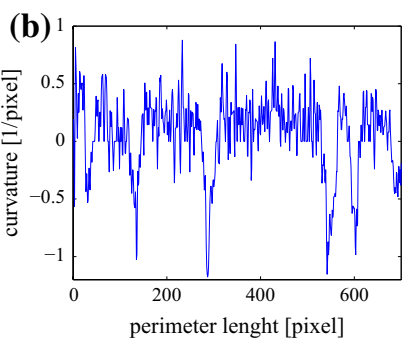

(d)

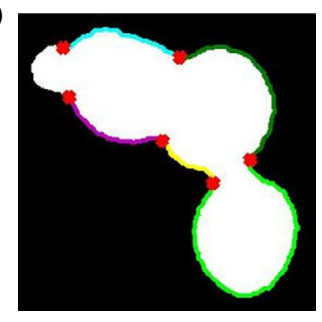

Fig. 6 Boundary detection and segmentation: a boundary along the object, $\mathbf{b}$ unfiltered curvature, $\mathbf{c}$ filtered curvature, $\mathbf{d}$ segmented boundary and curvature peaks

An example of a full-scale image processed with the above-described routine is presented in Fig. 7c.

\section{Code validation and error sources}

\subsection{Sample size}

It is indispensable to determine the sample size before conducting the experiment to ensure a certain confidence level and to minimize computation time. In this work, the sample size was determined by bootstrapping, a simple re-sampling method introduced by Efron (1979). Therefore, 6000 images were acquired. Out of the 6000 images, a sample of $p$ images is drawn without replacement and the mean bubble diameter is calculated. This procedure is repeated $k$ times, and the standard deviation of the $k$ mean values is calculated. For a sample size $p=500$ images and a repetition $k=200$, it was found that the coefficient of variation $\sigma\left(d_{\mathrm{b}, \text { mean }}\right) / \bar{d}_{\mathrm{b} \text {,mean }}$ is smaller than $0.5 \%$. A reasonable confidence level is reached (Bros and Cowell 1987).

\subsection{In-focus and out-of-focus bubbles}

In-focus and out-of-focus bubbles are recorded in shadow images. The boundary of the in-focus bubbles is sharper than the boundary of out-of-focus bubbles. This welldefined boundary of in-focus bubbles can be well determined, where the boundary of out-of-focus bubbles is always a bit blurred. The influence of this blur on the accuracy of size determination must be investigated.

(a)

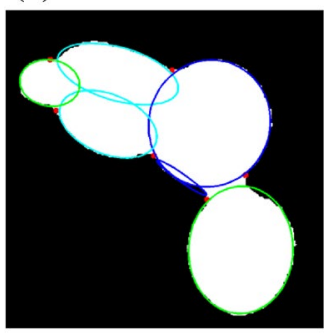

(b)

(c)

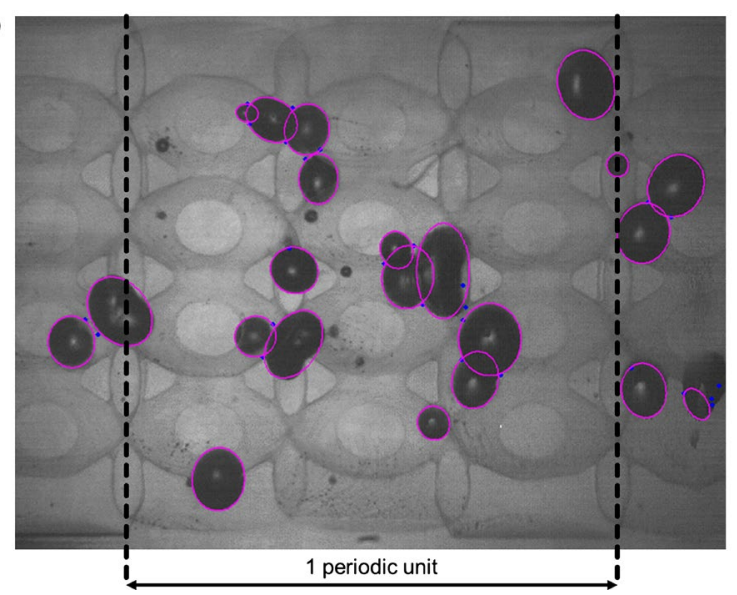

Fig. 7 Ellipse fitting: a without additional fitting criteria, b with additional fitting criteria, $\mathbf{c}$ example of fitted ellipse over one periodic unit

Therefore, a dot of $2.5 \mathrm{~mm}$ in diameter is plotted on a transparent foil and placed between background illumination and camera at three different locations. The results for out-of-focus and in-focus measurements are compared, and the results are summarized in Table 3. A maximum error of $9.4 \%$ is found for objects closer to the camera. Generally, the dimensions of out-of-focus bubbles are overestimated due to the blur.

It must be kept in mind that the foil is placed at $\pm 10 \mathrm{~mm}$. In the experiments, the largest diameter can never be at these locations as there is the wall of the pipe. Therefore, the bubbles are closer to the focal plane, and images of the bubbles are less blurred allowing an accurate determination of the dimensions. An image acquired in the real measurement campaign showing in- and out-of-focus bubbles is presented in Fig. 8.

\subsection{Code validation}

\subsubsection{Generation of synthetic data}

The developed routine for bubble detection and ellipse fitting was tested and verified using synthetic bubble swarms inside a tube of size $d=450$ pix and $h=600$ pix. Therefore, ellipsoids of random size, position and orientation 
Table 3 Error estimation of out-of-focus bubbles

\begin{tabular}{lll}
\hline Location & Size (pix) & Error $(\%)$ \\
\hline In-focus & 145.5 & Benchmark \\
$-10 \mathrm{~mm}$ & 159.2 & 9.4 \\
$+10 \mathrm{~mm}$ & 148.8 & 2.3 \\
\hline
\end{tabular}

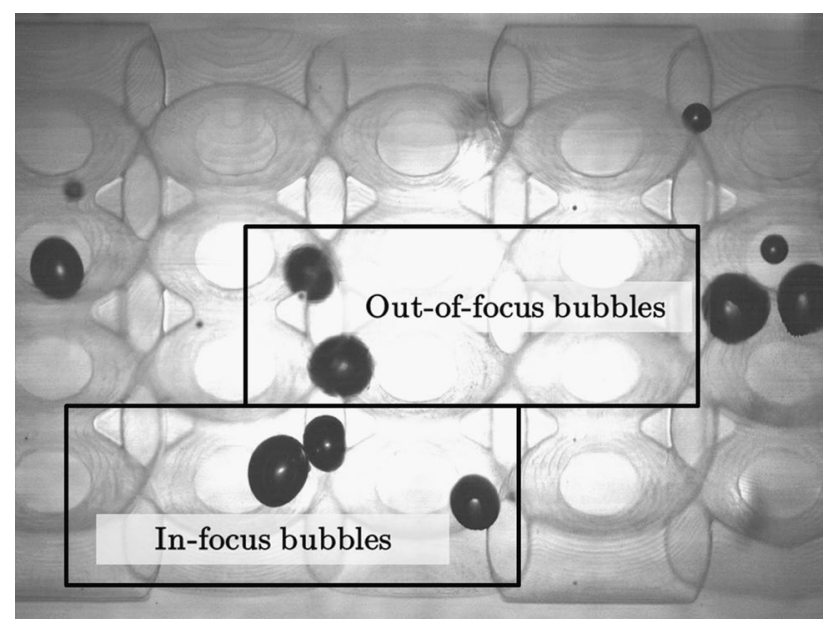

Fig. 8 Raw image showing in- and out-of-focus bubbles in the structure for a real measurement

Table 4 Simulation parameters for synthetic data

\begin{tabular}{lllll}
\hline & & Small & Medium & Large \\
\hline $\mathrm{a}_{\text {mean }}$ & (pix) & 20 & 30 & 40 \\
$\sigma_{a}$ & $($ pix $)$ & 10 & 10 & 10 \\
$a_{\text {min }}$ & $($ pix $)$ & 7 & 10 & 15 \\
$d_{\text {b,mean }} / d_{\text {pipe }}$ & $(-)$ & 0.0858 & 0.1209 & 0.1525 \\
\hline
\end{tabular}

were generated to investigate the effect of overlapping shadows and bubble's axis orientation. The major semi-axis $a$ of the ellipsoids is designed to lay within a normal distribution of the measured values. Table 4 shows the simulation parameters of the major semi-axis $a$. The minor semiaxes $b(0.65 \cdot a<b<1 \cdot a)$ and $c(0.85 \cdot a<c<1.15 \cdot a)$ are dependent on the major semi-axis $a$ and uniformly distributed inside the given intervals.

Small, medium and large bubbles have been generated to check the influence of the bubble size on the detection algorithm. The real bubble size measured in the experiments inside the structure $\left(d_{\mathrm{b} \text {,mean }} / d_{\text {pipe }}=0.061-0.1114\right)$ is in the range between small- and medium-sized synthetic bubbles. Therefore, the decision is made to further discuss the error for small bubbles. Figure 9 shows synthetic images at hold-ups of $0.5,2.5$ and $4.5 \%$. A total of 1500 images per hold-up were analyzed. (a)

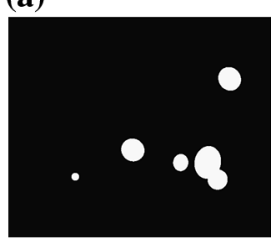

(b)

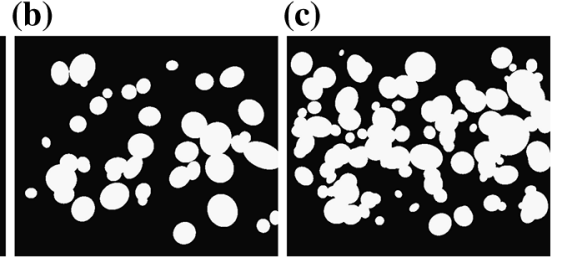

Fig. 9 Synthetic data of small bubbles, hold-up variation: a $\varepsilon=0.5 \%, \mathbf{b} \varepsilon=2.5 \%, \mathbf{c} \varepsilon=4.5 \%$

\subsubsection{Evaluation of synthetic data}

The projection of the ellipsoidal bubbles results in bubble shadow images. From this two-dimensional images, the diameter, surface area and volume of the original bubble should be determined. The challenge is that it is not known from a shadow image whether the original bubble is prolate or oblate. For the calculation of the bubble diameter, volume and surface area, different formulas are applicable, depending on the bubble shape. Either the quantities are calculated by a spherical approach where the major and the minor semi-axes are weighed equally, see Eqs. 6-8, or an ellipsoidal approach is used with one semi-axis being weighed more, see Eqs. 9-11 for oblate and Eqs. 12-14 for prolate ellipsoids.

$d_{\mathrm{b}, \mathrm{sp}}=2 \sqrt{a \cdot b}$

$V_{\mathrm{b}, \mathrm{sp}}=\frac{4}{3} \pi \cdot a^{3 / 2} \cdot b^{3 / 2}$

$A_{\mathrm{b}, \mathrm{sp}}=\pi \cdot d_{\mathrm{b}, \mathrm{sp}}{ }^{2}=4 \pi \cdot a \cdot b$

$d_{\mathrm{b}, \mathrm{o}}=2\left(a^{2} \cdot b\right)^{1 / 3}$

$V_{\mathrm{b}, \mathrm{o}}=\frac{4}{3} \pi \cdot a^{2} \cdot b$

$A_{\mathrm{b}, \mathrm{o}}=2 \pi \cdot a^{2} \cdot\left(1+\frac{1-e^{2}}{2} \tanh ^{-1} e\right)$

$d_{\mathrm{b}, \mathrm{pr}}=2\left(a \cdot b^{2}\right)^{1 / 3}$

$V_{\mathrm{b}, \mathrm{pr}}=\frac{4}{3} \pi \cdot a \cdot b^{2}$

$A_{\mathrm{b}, \mathrm{pr}}=2 \pi \cdot b^{2} \cdot\left(1+\frac{a}{b \cdot e} \sin ^{-1} e\right)$

where $d_{b}(\mathrm{~mm})$ : bubble diameter, $a(\mathrm{~mm})$ : major semiaxis, $b(\mathrm{~mm})$ : minor semi-axis, $V_{b}\left(\mathrm{~mm}^{3}\right)$ : bubble volume, 


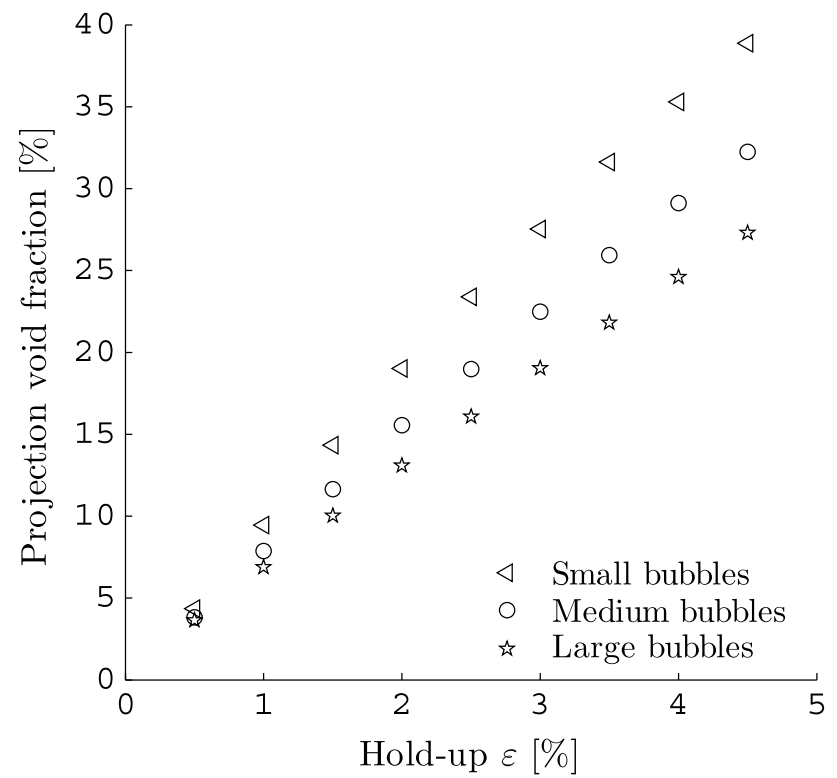

Fig. 10 Projection void fraction plotted versus the hold-up for the generated synthetic data

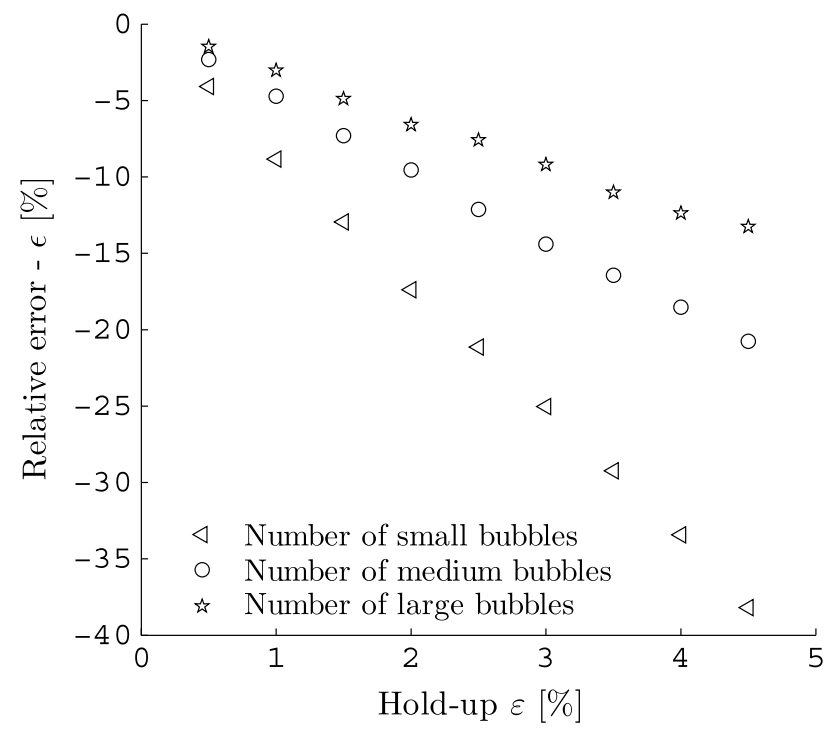

Fig. 11 Relative error of the number of bubbles (definition of bubble size, see Table 4)

$A_{b}\left(\mathrm{~mm}^{2}\right)$ : bubble surface and $e(-)$ : numerical eccentricity of the ellipsoid with $e^{2}=1-\frac{b^{2}}{a^{2}}$.

The values obtained from the bubble detection routine and Eqs. 6-14 are compared to the real quantities of the synthetic bubbles, and the relative error is calculated. Additionally, the projection void fraction is determined from the synthetic data and plotted versus the hold-up, see Fig. 10.

In Fig. 11, the relative error of the number of bubbles is plotted versus the hold-up. It can be seen that the number of bubbles is underestimated. The problem is the overlapping

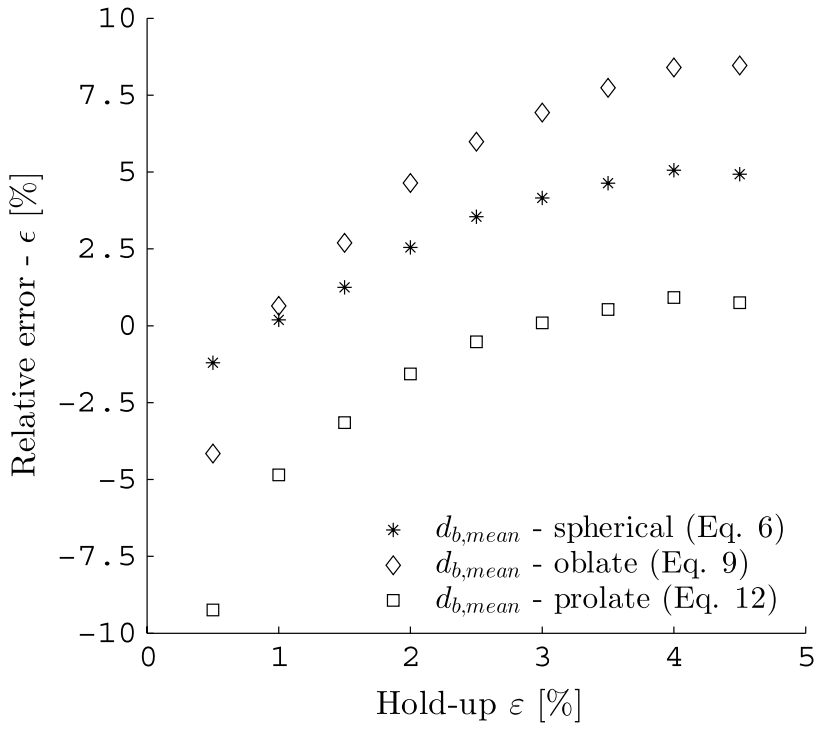

Fig. 12 Relative error of the arithmetic mean bubble diameter for small bubbles

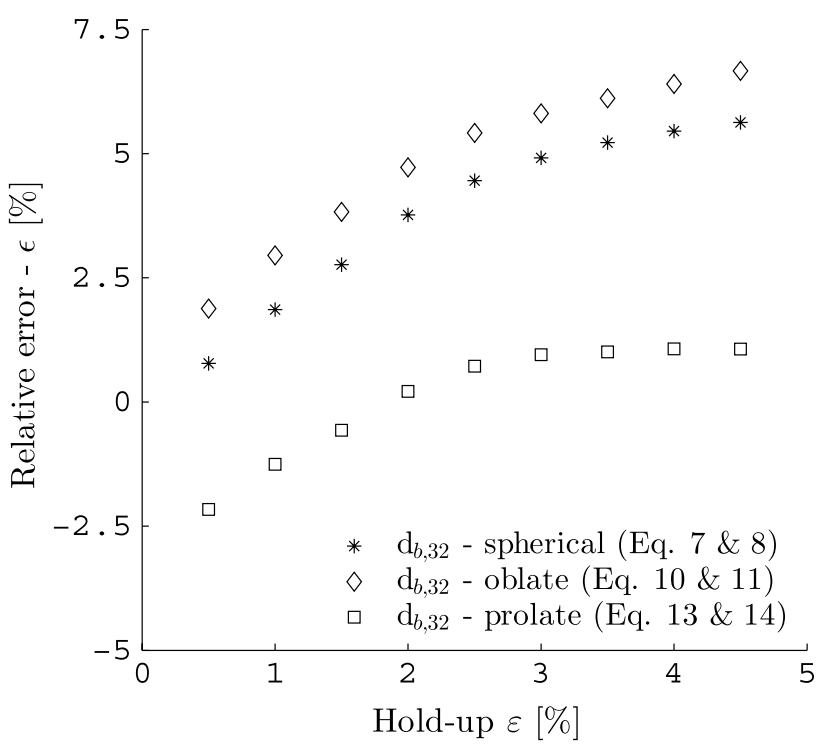

Fig. 13 Relative error of the Sauter mean bubble diameter for small bubbles

of bubble shadows, where bubbles are hidden behind each other and stay undetected. This effect is increased at higher hold-ups as more bubbles are present. For smaller bubbles, the effect is stronger as the probability of shadow overlapping is higher due to an increased projection void fraction, see Fig. 10.

The overlapping not only decreases the number of detected bubbles, but increases the measured diameter. Figure 12 shows the relative error of the arithmetic mean diameter plotted versus the hold-up for small bubbles. Overestimation of the diameter occurs for bubble shadows 


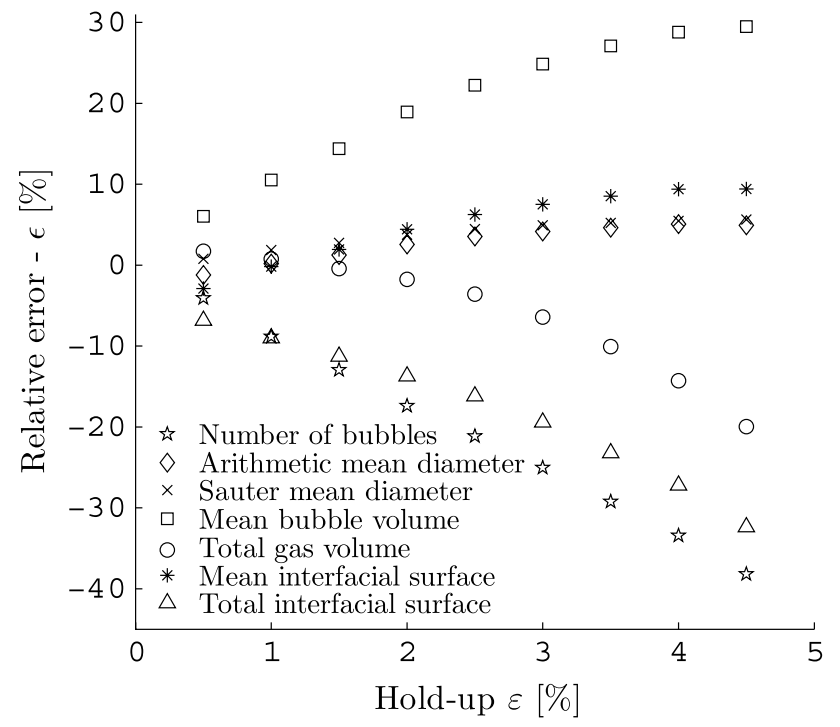

Fig. 14 Relative error of all quantities for the spherical approach for small bubbles

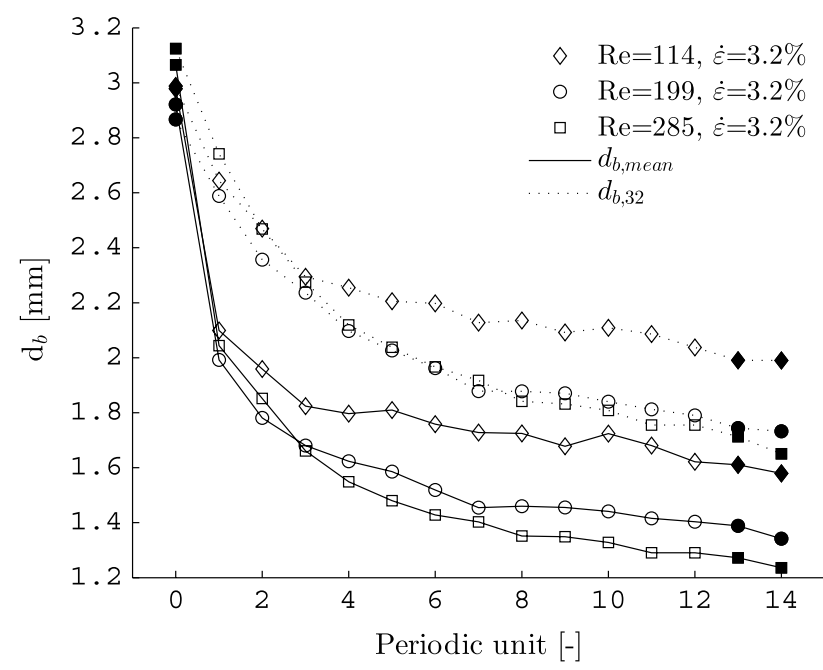

Fig. 15 Mean bubble diameter plotted along the porous structure

which are not completely overlapping, but overlapping as much as the detection routine cannot find any curvature peaks between two or more bubbles. Therefore, the ellipse is drawn around more than only one bubble increasing the diameter. At low hold-ups, the arithmetic mean bubble diameter is underestimated. This is explained by the fact that we cannot be sure to measure the major axis in the image. The major axis may be directed perpendicular to the image plane and stay hidden. In such cases, we only detect the two minor axes and underestimate the dimensions with all approaches.

Up to a hold-up of $2 \%$, the arithmetic mean diameter is best approximated by equally weighing the major and minor semi-axes. Therefore, the spherical approach for the calculation of the arithmetic mean diameter is used. The limitation of the hold-up at $2 \%$ is well justified as it represents the maximum hold-up in the measurements.

The result that the spherical approach is the most suitable can be explained by the fact that the investigated synthetic spheroids are close to spheres with a tendency to be prolate ellipsoidal. In reality, we also have rather round or slightly prolate ellipsoidal bubbles but never oblate ellipsoidal bubbles. Oblate ellipsoidal bubbles can be found in purely buoyancy-driven bubbly flows for rather large bubbles. In the streamed structure, spherical and prolate ellipsoidal bubbles are predominant due to the small bubble size and acceleration and deceleration effects.

Similarly to the arithmetic mean diameter, the Sauter mean diameter is determined and the relative error is plotted versus the hold-up, see Fig. 13. The Sauter mean diameter is best determined using the prolate approach. Nevertheless, up to a hold-up of $2 \%$, the spherical approach shows good results as well. With respect to all other quantities, it was decided to use the spherical approach to determining the Sauter mean diameter from the measurements.

The same analysis is carried out for the gas volume and interfacial area. The spherical approach delivers good results for all quantities up to a hold-up of $2 \%$.

Figure 14 is summarizing the results for the small bubbles evaluated with the spherical approach. The relative error is plotted versus the hold-up, including an estimation of the total gas volume and total interfacial area.

All averaged quantities, such as the arithmetic mean bubble diameter, Sauter mean diameter, mean bubble volume and mean interfacial area are only depending on the semi-axes but not on the total number of bubbles. The relative errors of these quantities are acceptable in the measurement range up to $2 \%$ hold-up. Maximum errors for the mean bubble surface are $-2.89 \%$ and for the mean bubble volume $18.93 \%$. It can be seen that the averaged quantities are generally overestimated due to the fact that the semi-axes are generally overestimated for highly overlapping bubbles, as explained above.

The global quantities, such as the total gas volume and total interfacial area are additionally depending on the number of bubbles. Therefore, the global quantities are generally underestimated as the number of bubbles is underestimated. The error range for a hold-up of up to $2 \%$ is given by $1.711 \%$ for the total gas volume and $-17.39 \%$ for the number of bubbles. These global quantities are very important as they are needed to determine the slip as well as the volume-specific interfacial area available for mass transport. Therefore, it is of great importance to accurately measure the total gas volume and total interfacial area. 


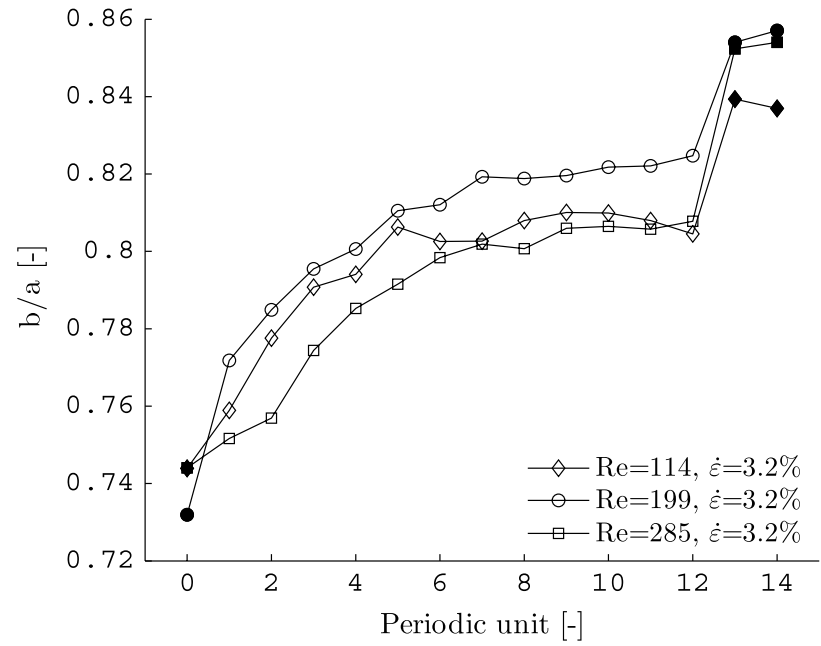

Fig. 16 Ratio of minor to major semi-axes (b/a) plotted along the porous structure

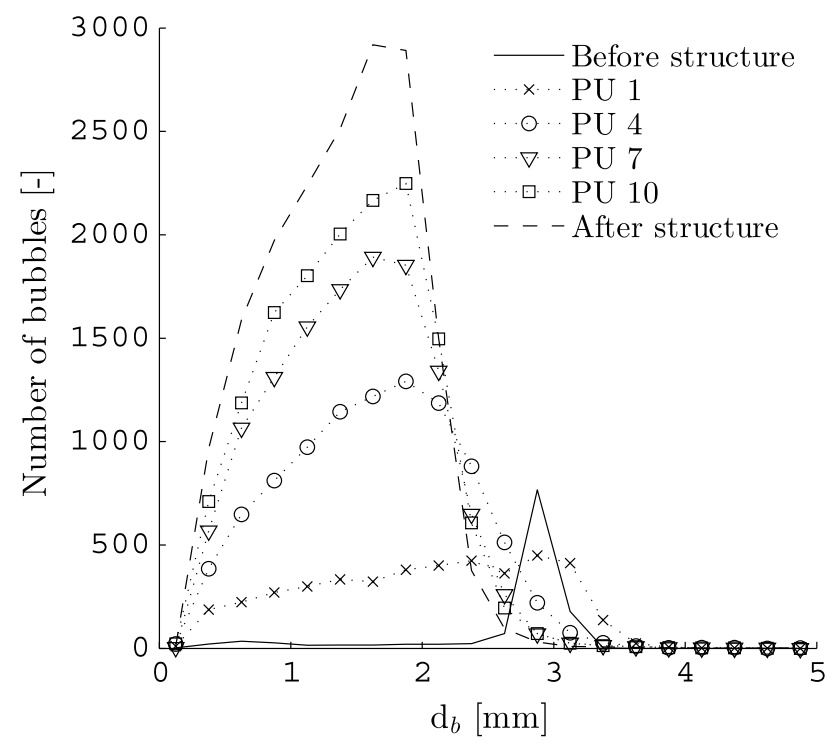

Fig. 17 Histogram of the bubble size, $R e=199, \varepsilon=3.2 \%$

Summarizing the code validation, it can be seen that all quantities can be determined with an accuracy of $\pm 20 \%$ at hold-ups of up to $2 \%$. The average-local-quantities are generally overestimated as they are only depending on the measured semi-axes. The global quantities are depending on the bubble size and the number of bubbles and are generally underestimated.

\section{Results}

The goal of this investigation is to determine global quantities, such as the total interfacial area, gas hold-up and slip.

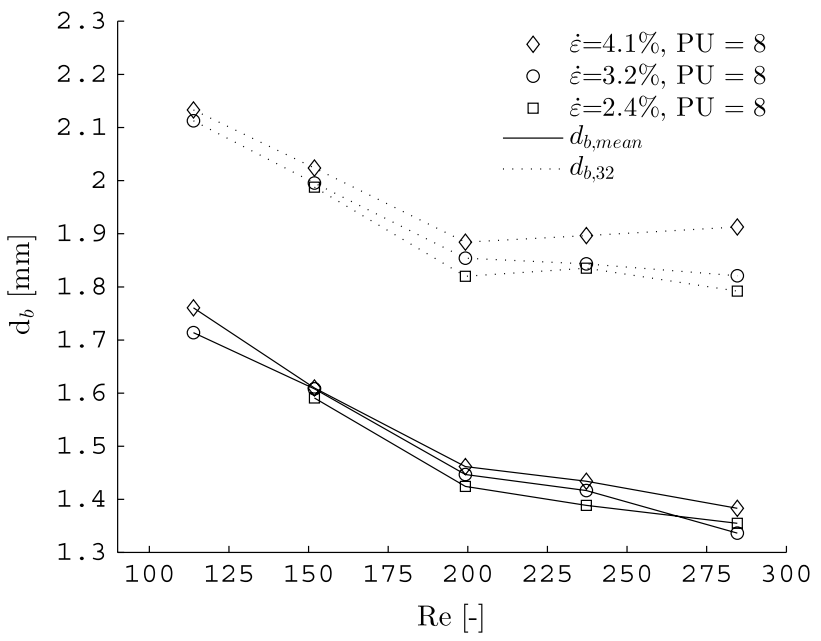

Fig. 18 Mean bubble diameter plotted versus the Reynolds number for three different volumetric transport fractions

Therefore, the number of bubbles and the bubble size are determined from the acquired images. In the following figures, the white markers indicate measurements inside the structure. The black markers indicate measurements up- and downstream of the structure in an empty tube of $20 \mathrm{~mm}$ in diameter.

\subsection{Bubble size, shape and size distribution}

In Fig. 15, the arithmetic mean diameter and the Sauter mean diameter are plotted along the porous structure. As expected, the Sauter mean diameter is larger than the arithmetic mean diameter. In the following, both mean diameters are called mean bubble diameter if the results are the same for both definitions. The mean bubble diameter at the inlet is about $3 \mathrm{~mm}$ for the three investigated Reynolds numbers. It can be seen that bubbles are mainly broken up in the first four to six periodic units. For higher Reynolds numbers, the mean bubble diameter at the outlet of the structure is smaller. Acceleration, deceleration and the corresponding shear stresses, turbulent eddies as well as the wall contact of bubbles are assumed to have the main influence on the bubble break-up.

The decrease in the bubble diameter at the exit of the structure (PU $=12-14)$ can be explained by the shape of the bubbles. Bubbles at the outlet have a more uniform and spherical shape, where inside the structure, the ratio of minor to major semi-axis is smaller. Figure 16 shows the ratio of minor to major semi-axis along the porous structure. It can be seen that the ratio of $b / a$ is increased along the structure. Especially at the outlet, a significant step can be observed. In the calculation of the mean diameter, the semi-axes are weighed equally. Therefore, the mean diameter is slightly overestimated for spheroids where $b / a$ is small. 


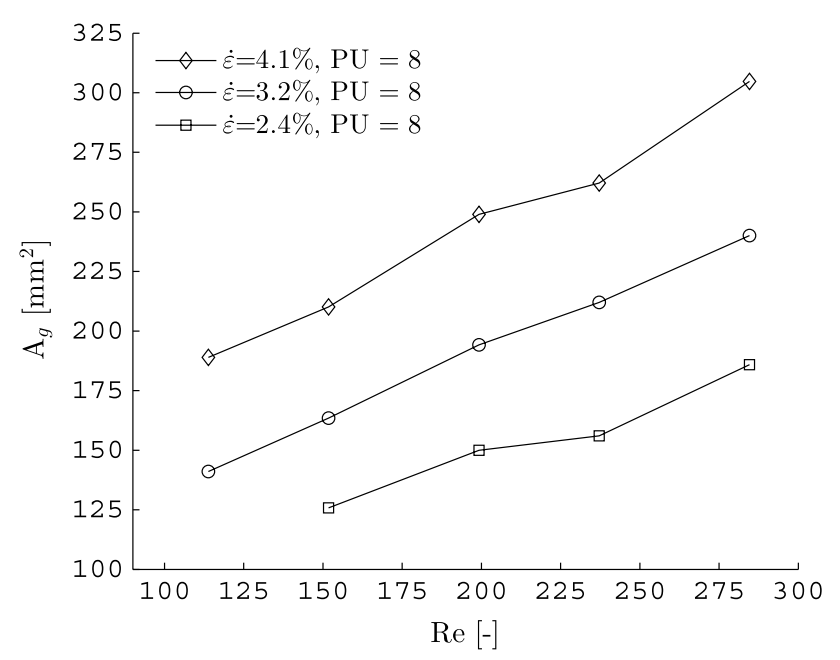

Fig. 19 Total interfacial area in periodic unit 8 plotted versus the Reynolds number

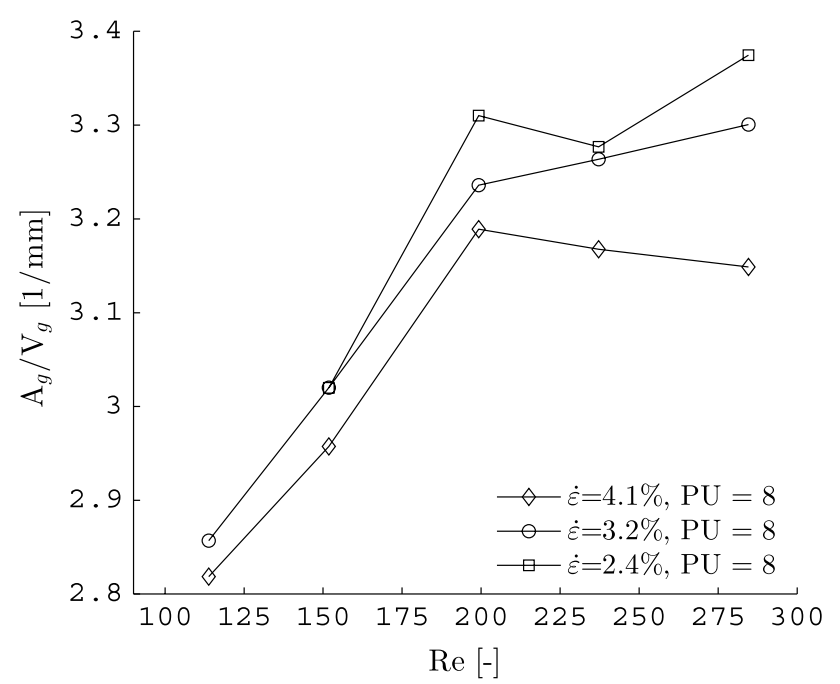

Fig. 20 Specific interfacial area in periodic unit 8 plotted versus the Reynolds number

Another reason for the drop of the mean diameter may be the bubble size distribution-related rising velocity. Larger bubbles will flow faster at the outlet, as the buoyancy force has a much larger influence and is not hindered by the structure. Therefore, more smaller bubbles are captured per image as they rise slower than the larger bubbles.

For a better understanding of the bubble size evolution, a histogram is given in Fig. 17. The bubbles coming from the sparger have a very uniform diameter of about $3 \mathrm{~mm}$ and are broken up in the first periodic unit into bubbles of a wide size range. Further downstream the structure, the distribution is getting more narrow and the bubbles are collected in less size classes.
In the eighth periodic unit, the mean bubble diameter was investigated for five different Reynolds numbers and three hold-ups. The eighth periodic unit is chosen as the flow is developed at this point and it is not too close to the exit. In Fig. 18, the mean diameter is plotted versus the Reynolds number. It can be seen that the main influence on the bubble size is coming from the fluid velocity. At higher Reynolds numbers, the bubbles are broken up into smaller bubbles. This is explained by the higher shear rates and velocity fluctuations.

Apart from the influence of the liquid velocity, the influence of the volumetric transport fraction is shown in Fig. 18. It can be seen that the influence is rather small. Nevertheless, it shows a larger mean diameter at higher volumetric transport fractions. This can be explained by the effect of an increased collision probability as more bubbles are in the structure. Higher collision probability will lead to more coalescence and therefore to larger bubbles.

\subsection{Interfacial area, hold-up and slip}

The main feature of the developed measurement technique lies in the detection and separation of overlapping bubble shadows inside complex geometries. It allows the determination of global quantities, such as the total interfacial area, hold-up or slip. In Fig. 19, the total interfacial area is plotted versus the Reynolds number.

The total interfacial area in the eighth periodic unit is increased with increasing liquid and gas flow rate. Two effects explain this behavior: (1) A higher gas flow rate must increase the total surface area if the bubble size is not increased. (2) Higher liquid flow rates increase the turbulence and therefore the bubble break-up.

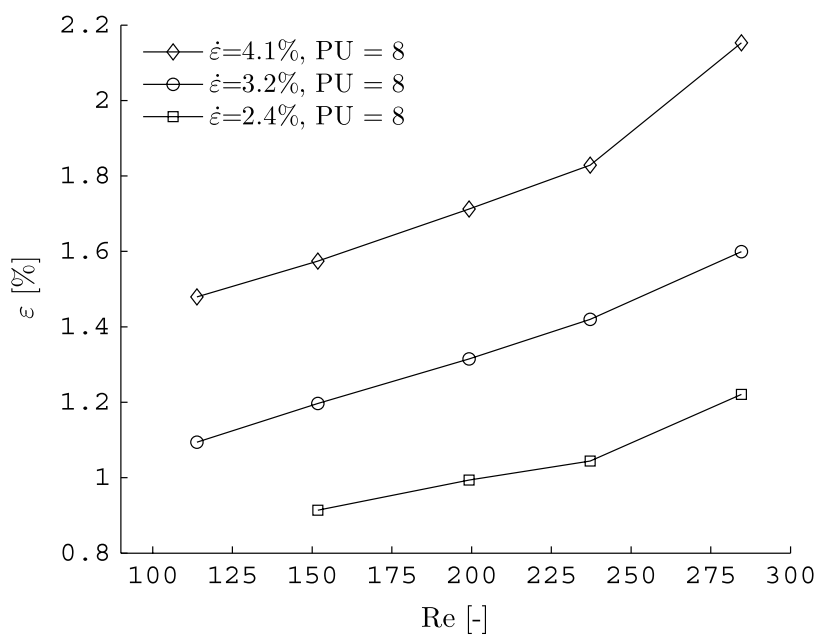

Fig. 21 Gas hold-up plotted versus the Reynolds number 


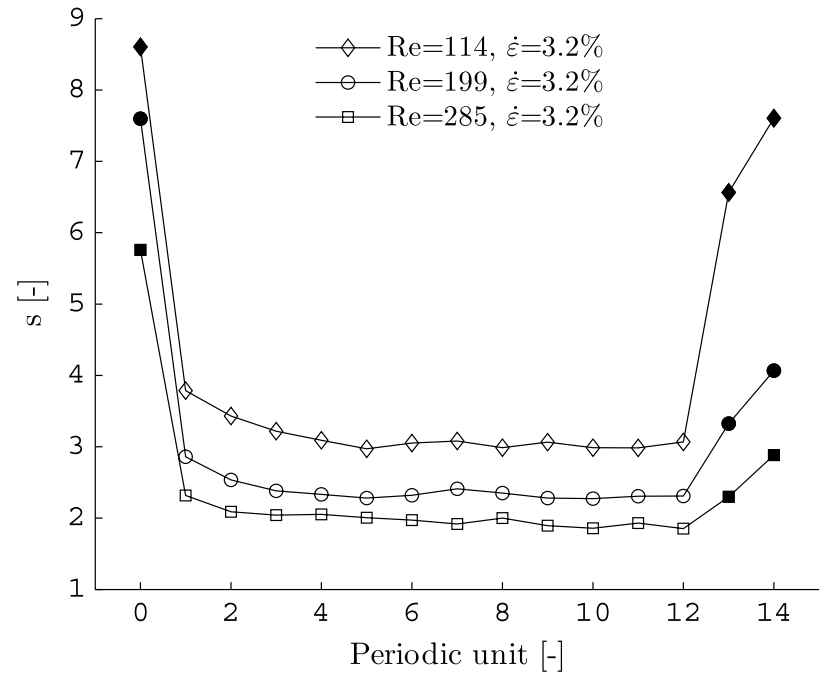

Fig. 22 Slip plotted along the porous structure

With respect to chemical reactions inside the structure, it is clear that a large interfacial area is of great interest for mass transport. Nevertheless, the information obtained from Fig. 19 is not sufficient. The specific interfacial area must be calculated relating the interfacial area to the gas volume.

Figure 20 shows the specific interfacial area plotted versus the Reynolds number. Now it is clear that the best conditions are given by lower volumetric transport fractions at medium Reynolds numbers. This is explained by the effect given in Sect. 5.1; at high volumetric transport fractions, bubbles will coalesce and form larger bubbles.

Such large bubbles cannot be broken up in flows with low Reynolds numbers as they do not bring enough energy into the system. For increasing fluid velocity, the interfacial area is increased for all volumetric transport fractions up to a Reynolds number of about 200. At high Reynolds numbers, the curve levels off and the specific interfacial area is not increased anymore. A similar change was seen in the mean diameter shown in Fig. 18. No clear explanation can be given as stated above. It is assumed that at higher turbulence the collision frequency and the corresponding coalescence rate is increased.

Summarizing the obtained information, it can be seen that a suitable Reynolds number and volumetric transport fraction must be found to optimize the operating conditions for every type of static mixer or tubular reactor. In this work, this optimized value is given at a rather low volumetric transport fraction at a Reynolds number of about 200 for the designed porous structure.

In Fig. 19, it can be seen that the interfacial area is depending on the volumetric transport fraction. To gain better insight, the hold-up is determined and plotted versus the Reynolds number shown in Fig. 21. It can be seen that the hold-up is increased for higher Reynolds numbers. The effect that the Reynolds number increases the hold-up at a constant volumetric transport fraction is explained by the lower slip between the phases. It shows that the volume is increased and the specific interfacial area stays constant from this point on.

Figure 21 also shows an increase in the hold-up for higher volumetric transport fractions. It is clear that for higher gas flow rates, the hold-up is increased too. The influence of the volumetric transport fraction on the holdup prevails compared to the influence coming from the liquid velocity.

Finally, the slip between gaseous and liquid phase is determined from the volumetric transport fraction and hold-up using Eq. 15. In Fig. 22, the slip is plotted along the structure.

$s=\frac{\dot{\varepsilon} \cdot(1-\varepsilon)}{\varepsilon \cdot(1-\dot{\varepsilon})}$

The slip right upstream the structure is much larger than in the first periodic unit, indicating that large bubbles inside the empty pipe gain a large part of their velocity from the buoyancy force. Once they reach the structure, the bubbles are broken up and the buoyancy force is reduced as the volume of the bubble is decreased. Additionally, the walls block the path of the bubble motion and slow down the bubbles movement. As soon as the bubble leaves the porous structure, this path is not blocked anymore and the bubble rising velocity is increased again, see periodic units 13-14. It also shows that the influence of the buoyancy force is more important than the velocity of the liquid phase at low Reynolds numbers. Generally, bubbles are slowed down inside the porous structure allowing a better transport of mass due to a longer residence time.

\section{Conclusion}

Shadow imaging is applied to a bubbly gas-liquid twophase flow inside a porous structure. Optical access is provided by index matching of the liquid phase and the structure material. The developed routine for bubble detection and sizing allows overlapping bubble shadows to be measured. It is shown that the developed technique is a valuable tool for volumetric two-phase flow measurements inside complex geometries. Important global quantities, such as gas hold-up or total interfacial area can be measured with only one camera. The accuracy is evaluated by running synthetic data. A maximum error range of $\pm 20 \%$ was determined for all quantities at hold-ups of up to $2 \%$.

It is shown that the specific interfacial area can be optimized by adjusting the volumetric transport fraction and 
the Reynolds number. A low volumetric transport fraction is good as the bubble collision probability is kept small. The collision probability is directly related to the coalescence of bubbles and therefore disadvantageous if the specific surface area must be increased for a better mass transfer.

On the one hand, the Reynolds number should be high enough to carry enough energy to break the bubbles. On the other hand, the Reynolds number should not be too large as the slip between the phases is decreasing. A decreased slip is responsible for a larger hold-up inside the structure. The collision probability is increased for a higher hold-up as more bubbles are present. Therefore, the mean diameter of the bubbles is increased and the specific interfacial area cannot be enlarged further.

Acknowledgments We gratefully acknowledge financial support from the Swiss Confederation's innovation promotion agency (CTI) in cooperation with DSM Nutritional Products.

\section{References}

Akhmetbekov YK, Alekseenko SV, Dulin VM, Markovich DM, Pervunin KS (2010) Planar fluorescence for round bubble imaging and its application for the study of an axisymmetric two-phase jet. Exp Fluids 48(4):615-629. doi:10.1007/s00348-009-0797-0

Belden J, Ravela S, Truscott TT, Techet AH (2012) Three-dimensional bubble field resolution using synthetic aperture imaging: application to a plunging jet. Exp Fluids 53(3):839-861. doi:10.1007/ s00348-012-1322-4

Bröder D, Sommerfeld M (2002) An advanced lif-plv system for analysing the hydrodynamics in a laboratory bubble column at higher void fractions. Exp Fluids 33(6):826-837. doi:10.1007/ s00348-002-0502-z

Bröder D, Sommerfeld M (2007) Planar shadow image velocimetry for the analysis of the hydrodynamics in bubbly flows. Meas Sci Technol 18(8):2513-2528. doi:10.1088/0957-0233/18/8/028

Bros WE, Cowell BC (1987) A technique for optimizing sample size (replication). J Exp Marine Biol Ecol 114(1):63-71. doi:10.1016/0022-0981(87)90140-7

Butscher D, Hutter C, Kuhn S, von Rohr PR (2012) Particle image velocimetry in a foam-like porous structure using refractive index matching: a method to characterize the hydrodynamic performance of porous structures. Exp Fluids 53(4):1123-1132. doi:10.1007/s00348-012-1346-9

Castanet G, Dunand P, Caballina O, Lemoine F (2013) High-speed shadow imagery to characterize the size and velocity of the secondary droplets produced by drop impacts onto a heated surface. Exp Fluids 54(3):1-17. doi:10.1007/s00348-013-1489-3

Chaouki J, Larachi F, Dudukovic MP (1997) Non-invasive monitoring of multiphase flows. Elsevier, Amsterdam. ISBN: 978-0-444-82521-6

Chen R, Fan LS (1992) Particle image velocimetry for characterizing the flow structure in three-dimensional gas-liquidsolid fluidized beds. Chem Eng Sci 47(13):3615-3622. doi:10.1016/0009-2509(92)85077-O

Clift R, Grace JR, Weber ME (2005) Bubbles, drops, and particles. Courier Dover Publications, Mineola
Colella D, Vinci D, Bagatin R, Masi M, ABu Bakr E (1999) A study on coalescence and breakage mechanisms in three different bubble columns. Chem Eng Sci 54(21):4767-4777. doi:10.1016/ S0009-2509(99)00193-1

DSM (2014) Product Data Sheet-Somos WaterShed XC 11122

Efron B (1979) Bootstrap methods: another look at the jackknife. Annals Stat 1-26

Fitzgibbon A, Pilu M, Fisher RB (1999) Direct least square fitting of ellipses. IEEE Trans Pattern Anal Mach Intell 21(5):476-480. doi:10.1109/34.765658

Fujiwara A, Danmoto Y, Hishida K, Maeda M (2004) Bubble deformation and flow structure measured by double shadow images and piv/lif. Exp Fluids 36(1):157-165. doi:10.1007/ s00348-003-0691-0

Garnier C, Lance M, Marié J (2002) Measurement of local flow characteristics in buoyancy-driven bubbly flow at high void fraction. Exp Thermal Fluid Sci 26(6):811-815. doi:10.1016/ S0894-1777(02)00198-X

Häfeli R, Altheimer M, Butscher D, Rudolf von Rohr P (2014) Piv study of flow through porous structure using refractive index matching. Exp Fluids 55(5):1-13. doi:10.1007/ s00348-014-1717-5

Häfeli R, Hutter C, Damsohn M, Prasser HM, Rudolf von Rohr P (2013) Dispersion in fully developed flow through regular porous structures: experiments with wire-mesh sensors. Chem Eng Process Process Intensif 69:104-111. doi:10.1016/j.cep.2013.03.006

Hibiki T, Ishii M (1999) Experimental study on interfacial area transport in bubbly two-phase flows. Int J Heat Mass Transf 42(16):3019-3035. doi:10.1016/S0017-9310(99)00014-9

Hirschberg S, Koubek R, Moser F, Schöck J (2009) An improvement of the sulzer smx static mixer significantly reducing the pressure drop. Chem Eng Res Design 87(4):524-532. doi:10.1016/j. cherd.2008.12.021

Honkanen M, Elcock D, Kuo CJ, Peles Y, Amitay M (2011) Lagrangian tracking of bubbles interacting with pin-fins in a microchannel. Exp Fluids 50(6):1527-1538. doi:10.1007/ s00348-010-1007-9

Honkanen M, Saarenrinne P, Stoor T, Niinimäki J (2005) Recognition of highly overlapping ellipse-like bubble images. Meas Sci Technol 16(9):1760. doi:10.1088/0957-0233/16/9/007

Hutter C, Mascarello F, Von Rohr PR, Ruppen D (2010) Device for processing and conditioning of material transported through the device. US Patent App. 13/376,266

Hutter C, Zenklusen A, Kuhn S, Rudolf von Rohr P (2011) Large eddy simulation of flow through a streamwise-periodic structure. Chem Eng Sci 66(3):519-529. doi:10.1016/j.ces.2010.11.015

Hutter C, Zenklusen A, Lang R, Rudolf von Rohr P (2011) Axial dispersion in metal foams and streamwise-periodic porous media. Chem Eng Sci 66(6):1132-1141. doi:10.1016/j.ces.2010.12.016

Kim JS, Kim SM, Kim HD, Ji HS, Kim KC (2012) Dynamic structures of bubble-driven liquid flows in a cylindrical tank. Exp Fluids 53(1):21-35. doi:10.1007/s00348-011-1224-x

Kitagawa A, Hishida K, Kodama Y (2005) Flow structure of microbubble-laden turbulent channel flow measured by piv combined with the shadow image technique. Exp Fluids 38(4):466-475. doi:10.1007/s00348-004-0926-8

Kong XZ, Holzner M, Stauffer F, Kinzelbach W (2011) Time-resolved $3 \mathrm{~d}$ visualization of air injection in a liquid-saturated refractiveindex-matched porous medium. Exp Fluids 50(6):1659-1670. doi:10.1007/s00348-010-1018-6

Lance M, Bataille J (1991) Turbulence in the liquid phase of a uniform bubbly air-water flow. J Fluid Mech 222:95-118. doi:10.1017/S0022112091001015

Lindken R, Merzkirch W (2000) Velocity measurements of liquid and gaseous phase for a system of bubbles rising in water. Exp Fluids 29(1):S194-S201. doi:10.1007/s003480070021 
Ma Y, Yan G, Scheuermann A, Bringemeier D, Kong XZ, Li L (2014) Size distribution measurement for densely binding bubbles via image analysis. Exp Fluids 55(12):1-6. doi:10.1007/ s00348-014-1860-Z

Murai Y, Matsumoto Y, Yamamoto F (2001) Three-dimensional measurement of void fraction in a bubble plume using statistic stereoscopic image processing. Exp Fluids 30(1):11-21. doi:10.1007/ s003480000129

Murakawa H, Kikura H, Aritomi M (2005) Application of ultrasonic doppler method for bubbly flow measurement using two ultrasonic frequencies. Exp Thermal Fluid Sci 29(7):843-850. doi:10.1016/j.expthermflusci.2005.03.002

Narrow T, Yoda M, Abdel-Khalik S (2000) A simple model for the refractive index of sodium iodide aqueous solutions. Exp Fluids 28(3):282-283. doi:10.1007/s003480050389

Nogueira S, Sousa R, Pinto A, Riethmuller M, Campos J (2003) Simultaneous piv and pulsed shadow technique in slug flow: a solution for optical problems. Exp Fluids 35(6):598-609. doi: 10.1007/s00348-003-0708-8

Northrup MA, Kulp TJ, Angel SM, Pinder GF (1993) Direct measurement of interstitial velocity field variations in a porous medium using fluorescent-particle image velocimetry. Chem Eng Sci 48(1):13-21. doi:10.1016/0009-2509(93)80279-Y
Otsu N (1975) A threshold selection method from gray-level histograms. Automatica 11(285-296):23-27

Rodríguez-Rodríguez J, Martínez-Bazán C, Montañes JL (2003) A novel particle tracking and break-up detection algorithm: application to the turbulent break-up of bubbles. Meas Sci Technol 14(8):1328. doi:10.1088/0957-0233/14/8/319

Sathe MJ, Thaker IH, Strand TE, Joshi JB (2010) Advanced piv/ lif and shadowgraphy system to visualize flow structure in two-phase bubbly flows. Chem Eng Sci 65(8):2431-2442. doi:10.1016/j.ces.2009.11.014

So S, Morikita H, Takagi S, Matsumoto Y (2002) Laser doppler velocimetry measurement of turbulent bubbly channel flow. Exp Fluids 33(1):135-142. doi:10.1007/s00348-002-0459-y

Svensson FJ, Rasmuson A (2006) Piv measurements in a liquid-liquid system at volume percentages up to $10 \%$ dispersed phase. Exp Fluids 41(6):917-931. doi:10.1007/s00348-006-0211-0

Willert C, Stasicki B, Klinner J, Moessner S (2010) Pulsed operation of high-power light emitting diodes for imaging flow velocimetry. Meas Sci Technol 21(7):075,402. doi:10.1088/0957-0233/21/7/075402

Wu Q, Ishii M (1999) Sensitivity study on double-sensor conductivity probe for the measurement of interfacial area concentration in bubbly flow. Int J Multiph Flow 25(1):155-173. doi:10.1016/ S0301-9322(98)00037-8 